\title{
Association of GSTM1, GSTT1, GSTM3, and GSTP1 Genes Polymorphisms with Susceptibility to Osteosarcoma: a Case- Control Study and Meta-Analysis
}

\author{
Mansour Moghimi ${ }^{1}$, Mohammad Reza Sobhan ${ }^{2 *}$, Mohammad Hossein \\ Jarahzadeh $^{3}$, Majid Morovati-Sharifabad ${ }^{4}$, Kazem Aghili ${ }^{5}$, Hossein Ahrar ${ }^{5}$, \\ Masoud Zare-Shehneh ${ }^{6}$, Hossein Neamatzadeh ${ }^{6}$
}

\begin{abstract}
Background: Some studies have investigated the association of GSTM1, GSTT1, GSTM3, and GSTP1 polymorphisms with susceptibility to osteosarcoma; however, these studies results are inconsistent and inconclusive. In order to drive a more precise estimation, the present case-control study and meta-analysis was performed to investigate association of GSTM1, GSTT1, GSTM3, and GSTP1 polymorphisms with osteosarcoma. Methods: Eligible articles were identified by a search of several electronic databases for the period up to May 5, 2018. Odds ratios were pooled using either fixed-effects or random effects models. Results: Finally, a total of 24 case-control studies with 2,405 osteosarcoma cases and 3,293 controls were included in the present meta-analysis. Overall, significantly increased osteosarcoma risk was found when all studies were pooled into the meta-analysis of GSTT1 (Null vs. Present: OR=1.247 95\% CI 1.020-1.524, $\mathrm{P}=0.031$ ) and GSTP1 polymorphism (B vs. A: OR= 8.899 95\% CI 2.722-29.094, P $\leq 0.001$ ). In the stratified, significantly increased osteosarcoma risk was observed for GSTT1 polymorphism among Asians (Null vs. Present: $\mathrm{OR}=1.300$ 95\% CI 1.034-1.635, $\mathrm{P}=0.025)$, but not among Caucasians. Conclusions: This meta-analysis demonstrated that GSTP1 and GSTT1 null genotype are associated with the risk of osteosarcoma. Future large welldesigned epidemiological studies are warranted to validate our results.
\end{abstract}

Keywords: Osteosarcoma- Glutathione-S-Transferase- polymorphism- meta-analysis

Asian Pac J Cancer Prev, 20 (3), 675-682

\section{Introduction}

Osteosarcoma is the most common primary malignant bone tumor (Mehdinejad et al., 2017; Sobhan et al., 2017), which characterized by the production of osteoid or immature bone due to the malignant proliferation of spindle stromal cells. The majority of osteosarcoma cases are sporadic that displays considerable heterogeneity and appears as various clinical entities showing a great span in tumor biology and prognosis (Calvert et al., 2012). The etiology of osteosarcoma is not well understood, however, recent studies suggest osteosarcoma cancers arise from primitive mesenchymal bone-forming cells. The best known example of an osteosarcoma predisposing factor is probably the exposure to self-luminous paint containing the radioactive substance radium, therapeutic radiation, and Paget disease. Osteosarcoma also occurs in certain genetic syndromes (in 7\% of cases) such as Li-Fraumeni syndrome (LFS), Werner Syndrome,
Rothmund Thomson Syndrome, Bloom Syndrome and Retinoblastoma (RB) (Calvert et al., 2012; Correa, 2016; Gorlick and Khanna, 2010; Savage and Mirabello, 2011). In a study showed that the childhood osteosarcoma has been associated with several parental malignancies such as colorectal, endocrine cancers, melanoma, and breast cancer (Ji and Hemminki, 2006).

Glutathione S-transferases (GSTs) are a major group of phase II metabolic enzyme that play an important role in the detoxification of certain endogenous compounds such as reactive oxygen species (ROS) or detoxification of xenobiotics including carcinogens, therapeutic drugs, and environmental pollutants (Dasaret al., 2017; Lushchak, 2012). The GSTs are polymorphic enzymes with inter-individual variations in enzymatic level and activity (Dasari et al., 2017). GSTM1, GSTT1 and GSTP1 are detoxification enzymes that have been known to metabolize a wide range of carcinogens. GSTM1 and GSTT1 gene are mapped to chromosome 1p13.3 and

${ }^{1}$ Department of Pathology, ${ }^{2}$ Department of Orthopedics, ${ }^{3}$ Department of Anesthesiology and Critical Care, ${ }^{5}$ Department of Radiology, ${ }^{6}$ Department of Medical Genetics, Shahid Sadoughi University of Medical Sciences, Yazd, ${ }^{4}$ Department of Basic Science, Faculty of Veterinary Medicine, Ardakan University, Ardakan, Iran.*For Correspondence: mrsobhanardakani@gmail.com 
22q11.23, respectively (Pejovic-Milovancevic et al., 2016). In both genes there is a deletion polymorphism that existing as null alleles, which results in the reduced activity of a functional gene product. It is reported that nearly $50 \%$ of the Caucasians carries a homozygous deletion of the GSTM1 gene (null genotype), resulting the lack of functional GSTM1 enzyme activity (Zheng et al., 2003). GSTM1 or GSTT1 gene deletions are associated with diseases such as infertility, rheumatoid arthritis, systemic sclerosis, Parkinson's disease, diabetes mellitus, and different of malignancies (Cao et al., 2017; Dahabreh et al., 2010; Ying et al., 2013; Zhaoet al., 2017). Other polymorphic genes of the GST family, in particular GSTM3 and GSTP1 have also been shown to be related to cancer risk modulation (Dahabreh et al., 2010; Ramsay and Dilda, 2014).

Many studies have shown that genetic variation in GSTs enzymes, including GSTP1 A313G (rs1695, Ile105Val) and homozygous deletions of the active alleles of GSTM1 and GSTT1 (null genotype) have a role in susceptibility to osteosarcoma (Barnette et al., 2004; Li et al., 2015; Lu et al., 2011; Peters et al., 2000; Qu et al., 2016; Salinas-Souza et al., 2010; Yang et al., 2012; Zhang et al., 2012). However, case-control studies undertaken in different populations gave controversial results, due to small sample size or other causes. Meta-analysis can be used to pool data from these studies to obtain sufficient statistical power to detect the potential effect of small to moderate sizes associated with these polymorphisms. Therefore, we conduct this meta-analysis to deal with these contradictory results and assess whether GSTM1, GSTT1, GSTM3, and GSTP1 polymorphisms contribute to the risk of osteosarcoma.

\section{Materials and Methods}

\section{Case-Control Study}

This case-control study was approved by the institutional Review Board of the Shahid Sadoughi University of Medical Sciences. In addition, all participants were voluntary and would complete the informed consent in written before taking part in this research. Fifty-one patients diagnosed with osteosarcoma were consecutively enrolled in this study between April 2014 and December 2016. All the included cases in this study were histologically confirmed. Sixty age ( \pm 4 years) and sex matched controls subjects who had no self-reported history of malignancies were recruited from the general population. Genomic DNA was isolated from peripheral blood leukocytes by using a Roche kit (Mannheim, Germany) in accordance with the manufacturer's protocol, and Extracted DNA was labeled and stored at $-20^{\circ} \mathrm{C}$ until used. Four selected GSTs polymorphisms (GSTM1, GSTT1, GSTM3, and GSTP1) were tested in this study. Genotyping was carried out using the Restriction Fragment Length Polymorphism of polymerase chain reaction (PCR-RFLP) method as described previously (Li et al. 2015; Qu et al. 2016).
Meta-Analysis

Identification and Eligibility of Relevant Studies

We searched electronic databases, including PubMed, EMBASE, ISI Web of Science, and Wanfang database of China, for all studies published up to the May 5, 2018, which had investigated the association of GSTM1, GSTT1, GSTM3, and GSTP1 polymorphisms with susceptibility to osteosarcoma. The following terms were used in various combinations in this search: ("osteosarcoma" OR "osteogenic sarcoma" OR "'sarcomas of bones") AND ("'glutathione S-transferase"' OR "GST") AND ("GSTM1" OR " GSTT1" OR "GSTM3" OR "GSTP1") AND ("polymorphism" OR "SNP" OR "'mutation" OR “variant" OR “variation"). References of the retrieved articles and review articles on this topic were also manually checked for additional relevant eligible studies. We selected all studies that had been published in English. If multiple reports were available for a same study population, we included only the most recent or largest case series.

\section{Inclusion and Exclusion Criteria}

The inclusion criteria were: (1) studies with case-control and cohort design; (2) studies that examined the association of GSTM1, GSTT1, GSTM3, and GSTP1 polymorphisms with osteosarcoma risk; and (3) studies with sufficient information for estimating odds ratio (OR) and their 95\% confidence interval (CI). The exclusion criteria were: (1) animal studies; (2) case only studies; (3) Abstracts, reviews, case reports, and commentaries; (4) studies did not report genotype frequencies or which the number of genotypes and alleles could not be ascertained; and (5) studies duplicate of previous publication or containing overlapping data. If studies had overlapping subjects, only the study with the largest population was finally selected. Additionally, we also checked for minor allelic frequency (MAF) among studies by different genotype frequencies in ethnic groups.

\section{Data Extraction}

The data from the published studies were extracted independently by two of the authors. For each study, the following variables were collected: first author's name, year of publication, country, ethnicity, sources of cases and controls, number of cases and controls, genotyping methods, allele numbers and genotype distributions in cases and controls, minor allele frequencies (MAFs) in control subjects, and the results of Hardy-Weinberg equilibrium (HWE) test. All disagreements about eligibility were resolved during a consensus meeting with a third reviewer. For studies including subjects of different ethnic groups, we extracted data separately for each ethnic group and categorized as Caucasian, Asian, African and others.

\section{Statistical Analysis}

The strengths of the association of GSTM1, GSTT1, GSTM3, and GSTP1 polymorphisms with susceptibility to osteosarcoma were assessed by calculating the pooled OR and its $95 \%$ confidence interval (CI). The significance of pooled ORs was determined by the Z-test. The pooled 
OR was calculated for dominant model, recessive model and allele comparisons. For meta-analysis of GSTM1 and GSTT1 polymorphisms, the pooled ORs were calculated using null vs. present model. For GSTP1 and GSTM3, the pooled ORs were calculated under five genetic models, i.e., allele (B vs. A), homozygote (BB vs. AA), heterozygote (BA vs. BB), dominant $(\mathrm{BB}+\mathrm{BA}$ vs. $\mathrm{AA})$ and recessive $(\mathrm{BB}$ vs. $\mathrm{BA}+\mathrm{AA})$. Between-study heterogeneities were estimated using the $\chi^{2}$-based Q-test and the heterogeneity was considered significant at $\mathrm{P}<0.05$. In addition, we used the $\mathrm{I}^{2}$ statistic to quantitatively evaluate heterogeneity $\left(\mathrm{I}^{2}<25 \%\right.$, low heterogeneity; $25 \% \leq \mathrm{I}^{2} \leq 75 \%$, moderate heterogeneity; $\mathrm{I}^{2}>75 \%$, high heterogeneity). When the heterogeneity was absent $\left(\mathrm{P}>0.05\right.$ or $\left.\mathrm{I}^{2}<50 \%\right)$, the fixed-effect model was used to pool the data from different studies; otherwise, the random-effects model (DerSimonian and Laird method) was applied. Hardy-Weinberg equilibrium (HWE) of the genotype frequencies in the control group was assessed by the goodness-of-fit $\chi^{2}$ test. Sensitivity analyses were conducted to identify the effect of individual study on pooled results and to test the reliability of results by deleting a single study each time. Studies not in HWE were subjected to a sensitivity analysis. To determine the presence of publication bias, both Begg's funnel plot (an asymmetrical funnel plot suggested a possible publication bias) and Egger's linear regression test were conducted, and $\mathrm{P}<0.05$ was considered significant. To explore the sources of cross-study heterogeneity, subgroup analysis by ethnicity was performed. All statistical analyses were performed using the CMA 2.0 software (Biostat, USA). Two-sided $\mathrm{P}<0.05$ was considered statistically significant.

\section{Results}

\section{Baseline Characteristics}

A flow chart of the literature search is shown in Figure 1. Finally, a total of 24 case-control studies in nine publications (Barnette et al., 2004; Li et al., 2015; Lu et al., 2011; Peters et al., 2000; Qu et al., 2016; Salinas-Souza et al., 2010; Yang et al., 2012; Zhang et al., 2012) including 2,405 osteosarcoma cases and 3,293 controls were included in the present meta-analysis. The characteristics of the 24 eligible studies are presented in Table 1 and 2. Of the studies included, nine studies with 875 cases and 1,245 controls concerned GSTM1, eight studies with 828 cases and 1,358 controls concerned GSTT1, three studies with 215 cases and 367 controls concerned GSTM3, and four studies with 487 cases and 323 controls concerned GSTP1. Of them, eight case-control studies were undertaken in Caucasians and 16 case-control studies in the Asians. All of the studies indicated that the distribution of genotypes in the controls was consistent with HWE except for two studies of GSTT1 (Table 2).

\section{Quantitative Data Synthesis GSTM1 and GSTT1 Null Polymorphisms}

Table 3 listed the main results of the meta-analysis of GSTM1 polymorphism and osteosarcoma risk. After the nine case-control studies were pooled into meta-analysis, no evidence of a significant association between GSTM1 polymorphisms and osteosarcoma risk was observed (Null vs. Present: $\mathrm{OR}=0.924 .95 \%$ CI $0.656-1.301, \mathrm{P}=0.651$, Figure 2). Similarly, no significant association was found in a subgroup analysis by ethnicity among Asians (Null

Table 1. Main Characteristics of Studies on GSTM1 and GSTT1 Included in This Meta-Analysis

\begin{tabular}{lllcccccc}
\hline First Author (Year) & Country (Ethnicity) & $\begin{array}{c}\text { Genotyping } \\
\text { Method }\end{array}$ & Case/ Control & \multicolumn{2}{c}{ Cases } & \multicolumn{2}{c}{ Controls } & MAFs \\
& & & Present & Null & Present & Null \\
\hline GSTM1 & & & & & & & \\
Peters, 2000 & Germany (Caucasian) & PCR-RFLP & $71 / 178$ & 41 & 30 & 85 & 93 & 0.261 \\
Barnette, 2004 & USA (Caucasian) & HTA & $12 / 236$ & 10 & 2 & 143 & 183 & 0.387 \\
Salinas-Souza, 2010 & Brazil (Caucasian) & PCR-RFLP & $80 / 160$ & 45 & 35 & 88 & 72 & 0.225 \\
Lu, 2011 & China (Asian) & TaqMan & $110 / 226$ & 49 & 61 & 122 & 104 & 0.23 \\
Yang, 2012 & China (Asian) & PCR-RFLP & $187 / 79$ & 95 & 92 & 42 & 37 & 0.234 \\
Zhang, 2012 & China (Asian) & TaqMan & $159 / 75$ & 67 & 92 & 39 & 36 & 0.24 \\
Li, 2015 & China (Asian) & Multi-PCR & $52 / 79$ & 36 & 16 & 42 & 37 & 0.234 \\
Qu, 2016 & China (Asian) & RFLP & $153 / 252$ & 88 & 65 & 157 & 95 & 0.188 \\
Present, 2018 & Iran (Asian) & PCR-RFLP & $51 / 60$ & 29 & 22 & 36 & 24 & 0.2 \\
GSTT1 & & & & & & & & \\
Peters, 2000 & Germany (Caucasian) & PCR-RFLP & $76 / 206$ & 55 & 16 & 159 & 47 & 0.114 \\
Barnette, 2004 & USA (Caucasian) & HTA & $12 / 300$ & 10 & 2 & 234 & 66 & 0.11 \\
Salinas-Souza, 2010 & Brazil (Caucasian) & PCR-RFLP & $80 / 160$ & 54 & 26 & 118 & 42 & 0.131 \\
Lu, 2011 & China (Asian) & TaqMan & $110 / 226$ & 40 & 70 & 115 & 111 & 0.244 \\
Yang, 2012 & China (Asian) & PCR-RFLP & $187 / 79$ & 69 & 118 & 32 & 47 & 0.297 \\
Zhang, 2012 & China (Asian) & TaqMan & $159 / 75$ & 54 & 105 & 31 & 44 & 0.293 \\
Qu, 2016 & China (Asian) & PCR-RFLP & $153 / 252$ & 85 & 68 & 145 & 107 & 0.212 \\
Present, 2018 & Iran (Asian) & PCR-RFLP & $51 / 60$ & 27 & 24 & 34 & 26 & 0.216 \\
\hline
\end{tabular}

HTA, High throughout assay; RFLP, Restriction fragment length polymorphism; MAF, Minor Allele Frequency. 
Table 2. Characteristics of Studies on GSTM3 and GSTP1 Included in the Meta-Analysis

\begin{tabular}{|c|c|c|c|c|c|c|c|c|c|c|c|c|c|c|c|}
\hline \multirow[t]{3}{*}{ First Author } & \multirow{3}{*}{$\begin{array}{l}\text { Country } \\
\text { (Ethnicity) }\end{array}$} & \multirow{3}{*}{$\begin{array}{l}\text { Genotyping } \\
\text { Technique }\end{array}$} & \multirow{3}{*}{$\begin{array}{l}\text { Case/ } \\
\text { Control }\end{array}$} & \multicolumn{5}{|c|}{ Cases } & \multicolumn{5}{|c|}{ Controls } & \multirow[t]{3}{*}{ MAFs } & \multirow[t]{3}{*}{ HWE } \\
\hline & & & & \multicolumn{3}{|c|}{ Genotypes } & \multicolumn{2}{|c|}{ Allele } & \multicolumn{3}{|c|}{ Genotypes } & \multicolumn{2}{|c|}{ Allele } & & \\
\hline & & & & AA & $\mathrm{AB}$ & BB & A & B & $\mathrm{AA}$ & $\mathrm{AB}$ & BB & A & B & & \\
\hline \multicolumn{16}{|l|}{ GSTM3 } \\
\hline Peters 2000 & $\begin{array}{l}\text { Germany } \\
\text { (Caucasian) }\end{array}$ & PCR-RFLP & $164 / 71$ & 116 & 47 & 1 & 279 & 49 & 48 & 19 & 4 & 115 & 27 & 0.19 & 0.269 \\
\hline Barnette 2004 & USA (Caucasian) & HTA & $12 / 236$ & 7 & 5 & 0 & 19 & 5 & 224 & 71 & 5 & 519 & 81 & 0.171 & 0.817 \\
\hline Present 2018 & Iran (Asian) & PCR-RFLP & $51 / 60$ & 34 & 16 & 1 & 84 & 18 & 38 & 21 & 1 & 97 & 23 & 0.191 & 0.315 \\
\hline GSTP1 & & & & AA & $\mathrm{AB}$ & BB & A & B & AA & $\mathrm{AB}$ & BB & A & B & & \\
\hline Yang 2012 & China (Asian) & PCR-RFLP & $187 / 71$ & 110 & 49 & 28 & 269 & 105 & 51 & 19 & 9 & 121 & 37 & 0.26 & 0.003 \\
\hline Zhang 2012 & China (Asian) & TaqMan & $159 / 75$ & 93 & 40 & 26 & 226 & 92 & 33 & 23 & 19 & 89 & 61 & 0.406 & 0.001 \\
\hline Qu 2016 & China (Asian) & PCR-RFLP & $90 / 117$ & 64 & 71 & 19 & 199 & 109 & 135 & 105 & 12 & 375 & 129 & 0.551 & 0.135 \\
\hline Present 2018 & Iran (Asian) & PCR-RFLP & $51 / 60$ & 32 & 16 & 3 & 80 & 22 & 39 & 19 & 2 & 97 & 23 & 0.192 & 0.864 \\
\hline
\end{tabular}

HTA, High throughout assay; RFLP, Restriction fragment length polymorphism; MAF, Minor Allele Frequency; HWE, Hardy-Weinberg Equilibrium

vs. Present: $\mathrm{OR}=1.185 .95 \% \mathrm{CI} 0.954-1.473, \mathrm{P}=0.125)$ and Caucasians (Null vs. Present: $\mathrm{OR}=0.450 .95 \% \mathrm{CI}$ 0.163-1.245, $\mathrm{P}=0.124$, Table 3).

Table 3 also listed the main results of the meta-analysis of GSTT1 polymorphism and osteosarcoma risk. When all the eligible studies were pooled into the meta-analysis of GSTT1 polymorphism, significantly increased risk of osteosarcoma was observed (Null vs. Present: OR= $1.24795 \%$ CI $1.020-1.524, \mathrm{P}=0.031)$. When stratified by ethnicity, a significant association between GSTT1 polymorphism and increased risk of osteosarcoma was detected among Asians (Null vs. Present: OR=1.300 95\% CI 1.034-1.635, $\mathrm{P}=0.025)$, but not among Caucasians.

\section{GSTM3 and GSTP1 Polymorphisms}

Table 3 listed the main results of the meta-analysis of GSTM3 and GSTP1 polymorphisms and osteosarcoma risk. By pooling all the studies, the GSTM3 polymorphism was not associated with osteosarcoma risk under all five genetic models, i.e., allele (B vs. $A$ : $\mathrm{OR}=0.85695 \%$ CI $0.584-1.254, \mathrm{P}=0.424$ ), heterozygote (BA vs. BB: $\mathrm{OR}=1.02295 \%$ CI $0.648-1.513, \mathrm{P}=0.925)$, homozygote (BB vs. $\mathrm{AA}: \mathrm{OR}=0.46995 \% \mathrm{CI} 0.104-2.108, \mathrm{P}=0.323$ ), dominant $(\mathrm{BB}+\mathrm{BA}$ vs. $\mathrm{AA}: \mathrm{OR}=0.93395 \% \mathrm{CI}$ $0.600-1.452, \mathrm{P}=0.760)$ and recessive $(\mathrm{BB}$ vs. $\mathrm{BA}+\mathrm{AA}$ : $\mathrm{OR}=0.42395 \%$ CI $0.095-1.886, \mathrm{P}=0.259)$. We also examined the association between GSTP1 polymorphism and osteosarcoma risk, and the overall result showed that GSTP1 polymorphism significantly associated with

Table 3. Pooled ORs for the Association of GSTs Polymorphisms with Osteosarcoma Risk

\begin{tabular}{|c|c|c|c|c|c|c|c|c|c|c|}
\hline \multirow[t]{2}{*}{ Subgroup } & \multirow[t]{2}{*}{ Genetic Model } & \multirow[t]{2}{*}{ Type of Model } & \multicolumn{2}{|c|}{ Heterogeneity } & \multicolumn{4}{|c|}{ Odds Ratio (OR) } & \multicolumn{2}{|c|}{ Publication Bias } \\
\hline & & & $\mathrm{I}^{2}(\%)$ & $\mathrm{P}_{\mathrm{H}}$ & OR & $95 \% \mathrm{CI}$ & $\mathrm{Z}_{\mathrm{OR}}$ & POR & $P_{\text {Beggs }}$ & $\mathrm{P}_{\text {Eggers }}$ \\
\hline \multicolumn{11}{|l|}{ GSTM1 } \\
\hline Overall & Null vs. Present & Random & 66.68 & 0.002 & 0.924 & $0.656-1.301$ & -0.453 & 0.651 & 0.047 & 0.008 \\
\hline Asians & Null vs. Present & Fixed & 25.69 & 0.242 & 1.185 & $0.954-1.473$ & 1.534 & 0.125 & 0.452 & 0.211 \\
\hline Caucasians & Null vs. Present & Fixed & 82.13 & 0.004 & 0.45 & $0.163-1.245$ & -1.538 & 0.124 & 0.296 & 0.152 \\
\hline \multicolumn{11}{|l|}{ GSTT1 } \\
\hline Overall & Null vs. Present & Fixed & 0 & 0.698 & 1.247 & $1.020-1.524$ & 2.155 & 0.031 & 0.536 & 0.368 \\
\hline Asians & Null vs. Present & Fixed & 0 & 0.559 & 1.3 & $1.034-1.635$ & 2.242 & 0.025 & 0.806 & 0.991 \\
\hline Caucasians & Null vs. Present & Fixed & 0 & 0.561 & 1.088 & $0.717-1.649$ & 0.369 & 0.692 & 1 & 0.579 \\
\hline \multicolumn{11}{|l|}{ GSTM3 } \\
\hline \multirow[t]{5}{*}{ Overall } & B vs. A & Fixed & 0 & 0.648 & 0.856 & $0.584-1.254$ & -0.8 & 0.424 & 0.296 & 0.02 \\
\hline & BA vs. AA & Fixed & 0 & 0.68 & 1.022 & $0.648-1.513$ & 0.094 & 0.925 & 1 & 0.5 \\
\hline & BB vs. AA & Fixed & 42.61 & 0.175 & 0.469 & $0.104-2.108$ & -0.988 & 0.323 & 0.296 & 0.026 \\
\hline & $\mathrm{BB}+\mathrm{BA}$ vs. AA & Fixed & 0 & 0.694 & 0.933 & $0.600-1.452$ & -0.305 & 0.76 & 0.296 & 0.275 \\
\hline & $\mathrm{BB}$ vs. $\mathrm{BA}+\mathrm{AA}$ & Fixed & 31.83 & 0.231 & 0.423 & $0.095-1.886$ & -1.128 & 0.259 & 0.296 & 0.044 \\
\hline \multicolumn{11}{|l|}{ GSTP1 } \\
\hline \multirow[t]{5}{*}{ Overall } & B vs. A & Random & 90.07 & $\leq 0.001$ & 8.899 & $2.722-29.094$ & 3.617 & $\leq 0.001$ & 0.734 & 0.612 \\
\hline & BA vs. AA & Fixed & 38.69 & 0.18 & 1.111 & $0.833-1.483$ & 0.718 & 0.473 & 0.308 & 0.37 \\
\hline & BB vs. AA & Random & 77.12 & 0.004 & 1.381 & $0.526-3.627$ & 0.654 & 0.513 & 0.734 & 0.763 \\
\hline & $\mathrm{BB}+\mathrm{BA}$ vs. AA & Fixed & 22.36 & 0.277 & 1.339 & $0.845-2.121$ & 1.243 & 0.214 & 0.734 & 0.874 \\
\hline & $\mathrm{BB}$ vs. $\mathrm{BA}+\mathrm{AA}$ & Fixed & 60.02 & 0.057 & 1.117 & $0.735-1.698$ & 0.517 & 0.605 & 0.734 & 0.612 \\
\hline
\end{tabular}




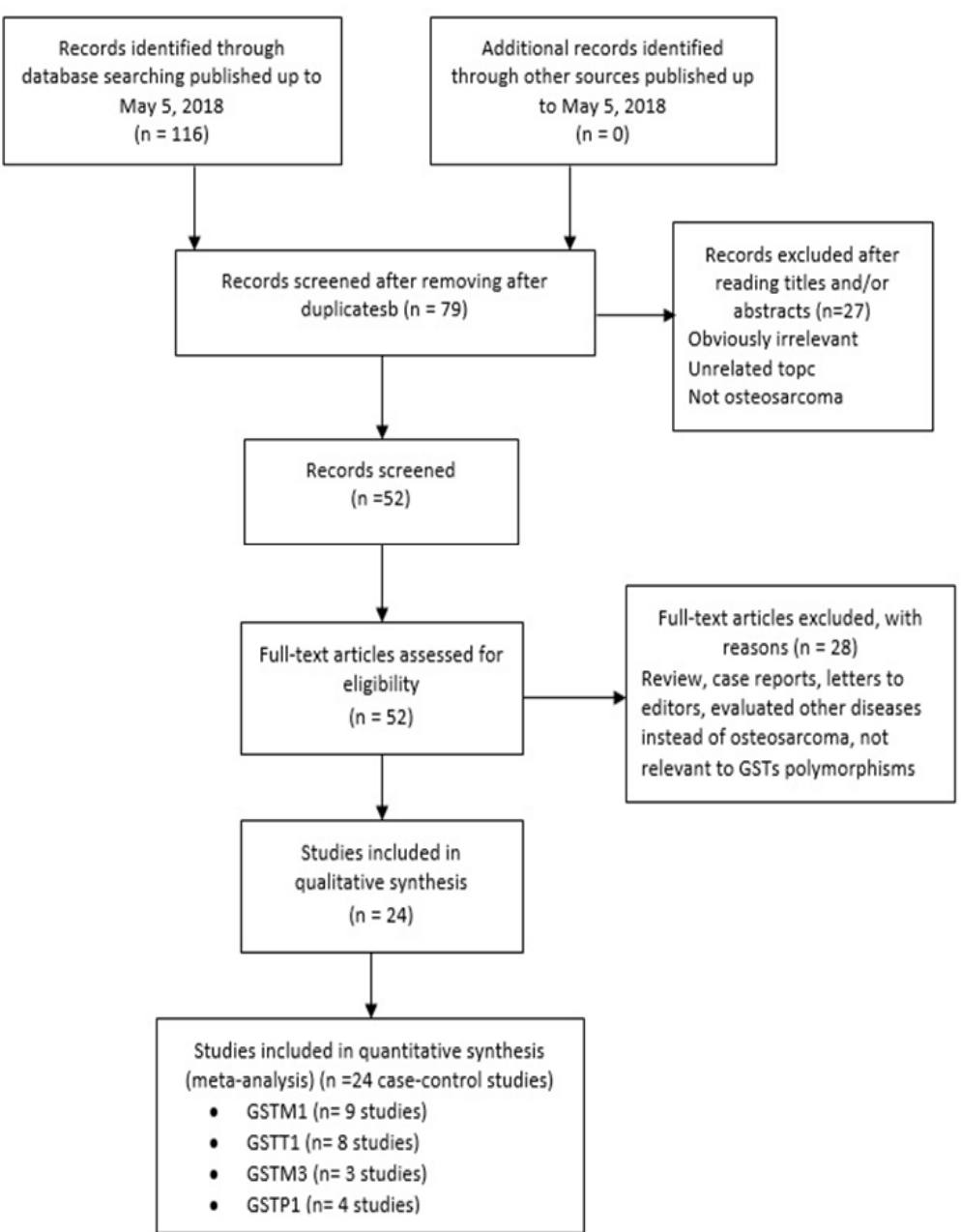

Figure 1. The study Selection and Inclusion Process

increased risk of osteosarcoma under the allele genetic model (B vs. A: OR= 8.899 95\% CI 2.722-29.094, $\mathrm{P} \leq 0.001$, Table 3).

\section{Publication Bias}

We used the Begg's funnel plot and Egger's in order to assess the publication bias of the literature. As shown in Figure 3, Begg's funnel plots did not reveal any evidence of obvious asymmetry in any of the models for GSTT1 and GSTP1 in the overall and subgroup analyses. Moreover, the results of the Egger's test also did not reveal any evidence of publication bias (Tables 3). However, there was a possible publication bias between GSTM1 polymorphism and osteosarcoma risk in the overall estimation $(\mathrm{PBeggs}=0.047$; PEggers $=0.008$, Figure 3 ). In addition, the Egger's test showed a significant publication bias between GSTP1 polymorphism and osteosarcoma risk in the overall estimation under three genetic models, i.e., allele $(\mathrm{PBeggs}=0.296$; Eggers $=0.020)$, homozygote $(\mathrm{PBeggs}=0.296$; PEggers $=0.026)$, and recessive $(\mathrm{PBeggs}$ $=0.296$; PEggers $=0.044$ ). Therefore, we used the Duval and Tweedie non-parametric "trim and fill" method to adjust for publication bias. Meta-analysis with and without "trim and fill" did not draw different conclusion, indicating that our results were statistically robust.

\section{Sensitivity Analysis}

Sensitivity analyses were performed to evaluate the effect of each study on the pooled ORs through sequential removal of individual studies. However, after removing the individual studies the pooled ORs did not significantly altered the pooled ORs for any of the four GSTs genes polymorphisms. Therefore, our results were relatively stable and credible (data not shown).

\section{Heterogeneity Analysis}

No significant between-study heterogeneity was observed for GSTT1 and GSTM3 polymorphisms in this meta-analysis. Although, a significant between-study heterogeneity was found for GSTM1 polymorphism in overall estimation $\left(\mathrm{I}^{2}=66.58 \%\right.$; $\left.\mathrm{PH}=0.002\right)$, and for GSTP1 polymorphism under two genetic models, allele $\left(\mathrm{I}^{2}=90.07 \% ; \mathrm{PH} \leq 0.001\right)$ and homozygote $\left(\mathrm{I}^{2}=77.12 \%\right.$; $\mathrm{PH}=0.004)$. 


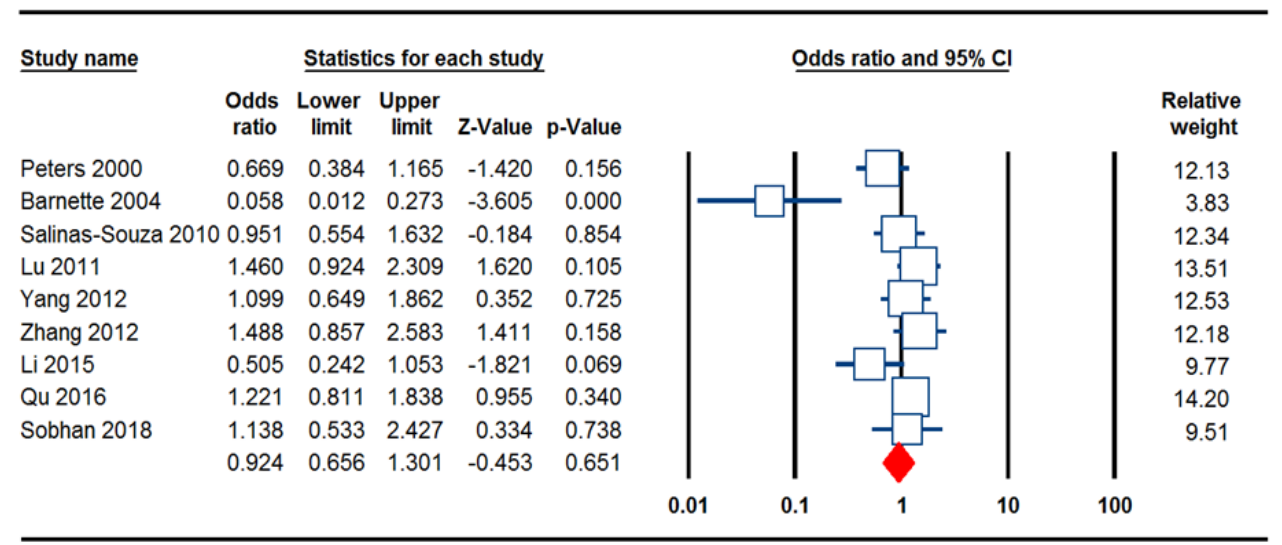

Figure 2. Forest Plot for Association of GSTM1 Null Genotype (Null vs. Present) with Osteosarcoma Risk in RandomEffects Model

\section{Discussion}

We conducted the meta-analysis to investigate the association of GSTM1, GSTT1, GSTM3, and GSTP1 polymorphisms with susceptibility to osteosarcoma. Overall, our meta-analysis indicates that GSTP1 and GSTT1 polymorphisms are associated with increased lung cancer risk when all eligible studies were pooled into the meta-analysis. Furthermore, subgroup analyses based on ethnicity found that the GSTT1 null genotype was at increased risk of osteosarcoma in Asian. However, we found no evidence of an association in Caucasians, indicating a difference in genetic diversity between different ethnic groups. It should be considered that the apparent inconsistency of these results may underlie differences in lifestyle and GSTs prevalence as well as possible limitations due to the relatively small sample size. Moreover, it is possible that the same SNP in GSTs genes may play different roles in osteosarcoma development, because osteosarcoma is a multifactorial and multi-genetic malignancy, and different genetic backgrounds may contribute to the discrepancy. Our results were consistent with the previous meta-analysis on GSTM1, GSTT1, GSTM3, and GSTP1 polymorphism and osteosarcoma risk (Wang et al., 2015). However, their findings about these polymorphisms and osteosarcoma risk essentially remains an open field, as the number of studies is considerably smaller than that needed to yield a robust conclusion.

Heterogeneity and publication bias are potentially significant problem when interpreting results of a meta-analysis (Jafari-Nedooshan et al., 2017; Namazi et al., 2015; Sadeghiyeh et al., 2017; Sobhan et al., 2017; Yazdi et al., 2017). In this meta-analysis, there was significant between-study heterogeneity for GSTM1 and GSTP1 polymorphisms, which might be due low quality studies such as HWE-violating studies. In addition, there was significant publication bias between GSTM1 and GSTP1 polymorphisms and osteosarcoma risk in this meta-analysis. This is a limitation for this meta-analysis because studies with null or negative findings, especially those with small sample size, are less likely to be published. However, adjusting for possible publication bias using the Duval and Tweedie nonparametric "trim and fill" method showed that the results did not change, indicating that the whole pooled results should be unbiased.

The present meta-analysis had several strengths. First, it was the biggest and most recent meta-analysis of the association of GSTM1, GSTT1, GSTM3, and GSTP1 polymorphisms with osteosarcoma risk; thus, it was more powerful than previous meta-analysis and case-control studies. Second, a systematic review of the

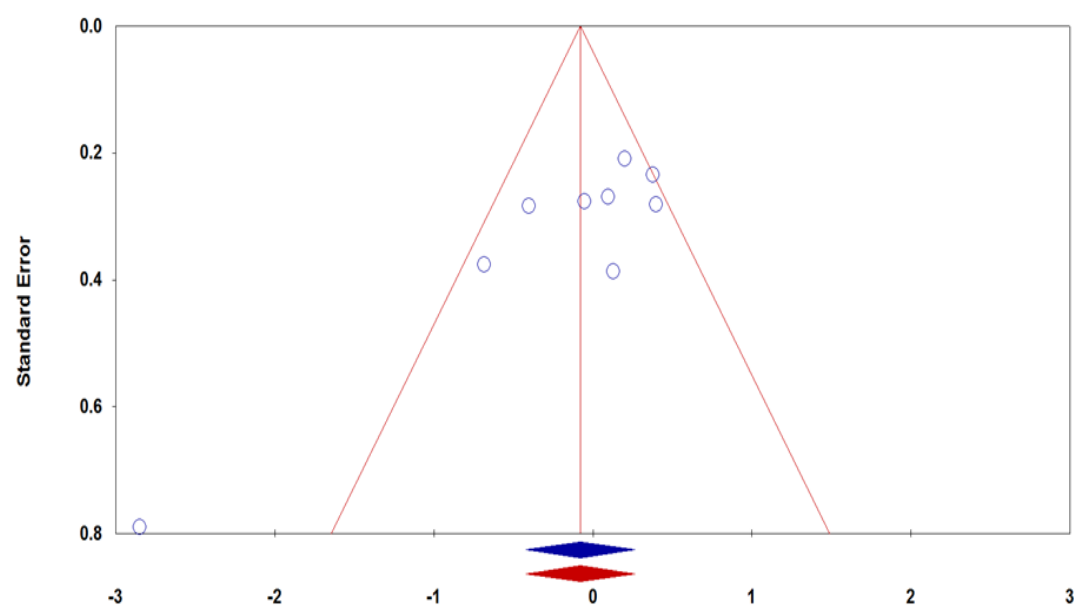

Figure 3. Funnel Plot for Publication Bias in the Meta-Analysis of GSTM1 Null Genotype (Null vs. Present) with Osteosarcoma Risk. Blue line without and red line with trim and fill test. 
association of GSTM1, GSTT1, GSTM3, and GSTP1 polymorphisms with osteosarcoma risk is statistically more powerful than any single study. Third, we have performed subgroup analysis by ethnicity for GSTM1 and GSTT1 null polymorphisms and indicated that the GSTT1 polymorphism was associated with osteosarcoma risk according to the patient ethnic background. Fourth, the pooled ORs for GSTs polymorphism are assessed under all five genetic models.

However, although we have put considerable efforts and resources into testing possible association of GSTs polymorphisms with osteosarcoma risk, there are still some limitations remained from the published studies, which should be addressed. First, although we have enrolled all the eligible studies in this meta-analysis, the sample size of the included studies was not large enough, which the results could be influenced by type I and type II errors. Thus, the results lacked statistical power and reliability to confirm or refute the association of GSTs polymorphisms with osteosarcoma risk in a definitive manner. In addition, the number of studies included in this meta-analysis was relatively small for GSTM3 and GSTP1, which makes it hard to perform subgroup analysis ethnicity. Second, all included articles were published in English. Therefore, unpublished studies and studies in other languages might be missed, which produced selection bias at the start of our study. Fourth, the overall outcomes were based on individual unadjusted ORs, while a more precise evaluation should be adjusted by other potentially suspected co-variants including age, sex, life style, and environmental factors. Finally, it is well known that osteosarcoma is a multifactor disease, and it is unlikely that a single variation in a gene would be obviously associated with an increase in osteosarcoma risk. But, we had insufficient data to evaluate the effects of SNP-SNP, gene-gene and gene-environment interactions were not addressed in this meta-analysis.

In conclusion, our study suggests that GSTP1 and GSTT1 null genotype may be associated with an increased risk of osteosarcoma. Moreover, no association with osteosarcoma risk is identified in GSTM1 and GSTM3 polymorphisms. Due to the limitations mentioned above, more comprehensive and large well-designed studies are warranted to validate the present findings.

\section{Funding}

No special grant or funding was used.

\section{Conflict of interest}

All authors declare no conflict of interest.

\section{References}

Barnette P, Scholl R, Blandford M, et al (2004). High-throughput detection of glutathione s-transferase polymorphic alleles in a pediatric cancer population. Cancer Epidemiol Biomarkers Prev, 13, 304-13.

Calvert GT, Randall RL, Jones KB,et al (2012). At-risk populations for osteosarcoma: The syndromes and beyond. Sarcoma, 2012, 1-9.

Cao T, Xu N, Wang Z, Liu H (2017). Effects of glutathione
S-transferase gene polymorphisms and antioxidant capacity per unit albumin on the pathogenesis of chronic obstructive pulmonary disease. Oxid Med Cell Longev, 2017, 6232397.

Correa H (2016). Li-fraumeni syndrome. J Pediatr Genet, 5, 84-8.

Dahabreh IJ, Giannouli S, Gota V, Voulgarelis M (2010). GSTT1 and GSTM1 polymorphisms and myelodysplastic syndrome risk: a systematic review and meta-analysis. Int $J$ Cancer, 126, 1716-23.

Dasari S, Lokanatha Oruganti HB, Balaji M (2017). Glutathione S-transferases detoxify endogenous and exogenous toxic agents- minireview. J Dairy Vet Anim Res, 5, 1-3.

Gorlick R, Khanna C (2010). Osteosarcoma. J Bone Miner Res, 25, 683-91.

Jafari-Nedooshan J, Kargar S, Neamatzadeh H, et al (2017). Lack of association of the fat mass and obesity associated (FTO) gene rs9939609 polymorphism with breast cancer risk: a systematic review and meta-analysis based on case - control studies. Asian Pac J Cancer Prev, 18, 1031-7.

Ji J, Hemminki K (2006). Familial risk for histology-specific bone cancers: An updated study in Sweden. Eur J Cancer, 42, 2343-9.

Li L, Li JG, Liu CY, Ding YJ (2015). Effect of CYP1A1 and GSTM1 genetic polymorphisms on bone tumor susceptibility. Genet Mol Res, 14, 16600-7.

Lu X-F, Yang W-L, Wan Z-H, Li J, Bi Z-G (2011). Glutathione S-transferase polymorphisms and bone tumor risk in China. Asian Pac J Cancer Prev, 12, 3357-60.

Lushchak VI (2012). Glutathione homeostasis and functions: potential targets for medical interventions. J Amino Acids, 2012, 1-26.

Mehdinejad M, Sobhan MR, Mazaheri M, et al (2017). Genetic association between ERCC2, NBN, RAD51 gene variants and osteosarcoma risk: A systematic review and meta-analysis. Asian Pacific J Cancer Prev, 18, doi:10.22034/APJCP.2017.18.5.1315.

Namazi A, Abedinzadeh M, Nourbaksh P, Neamatzadeh H (2015). Association between the XRCC3 Thr241Met polymorphism and risk of colorectal cancer: A meta analysis of 5,193 cases and 6,645 controls. Asian Pac J Cancer Prev, 16, doi:10.7314/APJCP.2015.16.6.2263.

Pejovic-Milovancevic MM, Mandic-Maravic VD, Coric VM, et al (2016). Glutathione S-transferase deletion polymorphisms in early-onset psychotic and bipolar disorders: A case-control study. Lab Med, 47, 195-204.

Peters U, Preisler-Adams S, Hebeisen A, et al (2000). Glutathione S-transferase genetic polymorphisms and individual sensitivity to the ototoxic effect of cisplatin. Anticancer Drugs, 11, 639-43.

Qu WR, Wu J, Li R (2016). Contribution of the GSTP1 gene polymorphism to the development of osteosarcoma in a Chinese population. Genet Mol Res, 15, doi:10.4238/ gmr.15038034.

Ramsay EE, Dilda PJ (2014). Glutathione S-conjugates as prodrugs to target drug-resistant tumors. Front Pharmacol, 5,181 .

Sadeghiyeh T, Hosseini Biouki F, Mazaheri M, et al (2017). Association between catechol-O-methyltransferase val158Met (158G/A) polymorphism and suicide susceptibility: A meta-analysis. J Res Health Sci, 17, e00383.

Salinas-Souza C, Petrilli AS, de Toledo SRC (2010). Glutathione S-transferase polymorphisms in osteosarcoma patients. Pharmacogenet Genomics, 20, 507-15.

Savage SA, Mirabello L (2011). Using epidemiology and genomics to understand osteosarcoma etiology. Sarcoma, 2011, 1-13.

Sobhan MR, Yazdi MF, Mazaheri M, Shehneh MZ, Neamatzadeh 
$\mathrm{H}$ (2017). Association between the DNA repair gene XRCC3 rs861539 polymorphism and risk of osteosarcoma: A systematic review and meta-analysis. Asian Pacific J Cancer Prev, 18, doi:10.22034/APJCP.2017.18.2.549.

Sobhan MR, Mehdinejad M, Jamaladini MH,et al (2017). Association between aspartic acid repeat polymorphism of the asporin gene and risk of knee osteoarthritis: A systematic review and meta-analysis. Acta Orthop Traumatol Turc, 51, doi:10.1016/j.aott.2017.08.001.

Wang Z, Xu H, He M, et al (2015). The association of glutathione S-transferase polymorphisms in patients with osteosarcoma: evidence from a meta-analysis. Eur J Cancer Care (Engl), 24, 417-24.

Yang L-M, Li X-H, Bao C-F (2012). Glutathione S-transferase $\mathrm{P} 1$ and DNA polymorphisms influence response to chemotherapy and prognosis of bone tumors. Asian Pac J Cancer Prev, 13, 5883-6.

Yazdi MM, Jamalaldini MH, Sobhan MR, et al (2017). Association of ESR $\alpha$ gene Pvu II T \&gt; C, XbaI A \&gt; G and BtgI G \&gt; A polymorphisms with knee osteoarthritis susceptibility: A systematic review and meta-analysis based on 22 case-control studies. Arch Bone Jt Surg, 5, doi:10.22038/abjs.2017.22391.1578.

Ying H-Q, Qi Y, Pu X-Y, Liu S-R, A Z-C (2013). Association of GSTM1 and GSTT1 genes with the susceptibility to male infertility: result from a meta-analysis. Genet Test Mol Biomarkers, 17, 535-42.

Zhang S-L, Mao N-F, Sun J-Y, et al (2012). Predictive potential of glutathione S-transferase polymorphisms for prognosis of osteosarcoma patients on chemotherapy. Asian Pac J Cancer Prev, 13, 2705-9.

Zhao E, Hu K, Zhao Y (2017). Associations of the glutathione S-transferase P1 Ile105Val genetic polymorphism with gynecological cancer susceptibility: a meta-analysis. Oncotarget, 8, 41734-9.

Zheng T, Holford TR, Zahm SH, et al (2003). Glutathione S-transferase M1 and T1 genetic polymorphisms, alcohol consumption and breast cancer risk. Br J Cancer, 88, 58-62.

This work is licensed under a Creative Commons AttributionNon Commercial 4.0 International License. 